\title{
II.
}

\section{Ueber die Veränderungen der Nervenelemente des Centralnervensystems bei der Hundswuth.}

\author{
Von Prof. N. M. Popoff in Warschau.
}

(Hierzu Taf. I.)

Die Anatomo-Pathologen haben schon öfters ihre Aufmerksamkeit auf die im Centralnervensystem während der Lyssa vorkommenden Veränderungen gerichtet, beschränkten sich aber bis vor Kurzem nur auf das Untersuchen derjenigen, welche bei diesem Leiden die Blutgefässe zur Licht bringen. Den Zustand der Nervenelemente erwähntén sie gewöhnlich gar nicht oder sehr oberflächlich.

Einer der ersten Forscher in dieser Hinsicht, Prof. Benedik $\left.\left.t^{1}\right)^{2}\right)^{3}$ ), kam auf Grund einer sehr detaillirten Analyse der im Gehirn und Rückenmark unter Wirkang des Hundswuthgiftes vorkommenden Erscheinungen zur Ueberzeugung, dass man hier als erstes Symptom das Gerinnen des Blutes in den Gefässen annehmen müsse. Solche Verstopfung eines ansehnlichen Theiles des Gefässsystems hat zur Folge eine Steigerung des Blutdruckes und Blutaustritte; die ausgetretene Flüssigkeit, welche besondere Eigenschafto besitat, durchdringt das umgebende Gewebe und verändert sich schliesslich mit letzterem in eine feinkörnige Masse. Die Heerde dieser Masse hält Prof. Benedikt für die wichtigsten, im Centralnervensystem bei der Lyssa vorkommenden Erscheinungen; eine Erwähnung der Nervenelemente zu machen, hält er für überflüssig, indem er durch diese Aenderungen das ganze Leidensbild erklärt.

Fast derselben Meinung in dieser Frage ist ein anderer Forscher, Kolessnik off $\left.{ }^{4}\right)^{5}$ ). In vielen Einzelheiten mit dem Vor-

1) Wiener med. Presse. 1874.

2) Zur patbolog. Anatomie der Lyssa. Dieses Archiv. 1875. Bd. 64.

3) Zur patholog. Anatomie der Lyssa. Dieses Archiv. 1878. Bd. 72.

4) Centralbl. f. d. med. Wissensch. 1875. No. 50 .

5) Ueber patholog. Veränderungen des Gehirns und Räckenmarks der Hunde bei der Lyssa. Dieses Archiv. 1881. Bd. 81. 

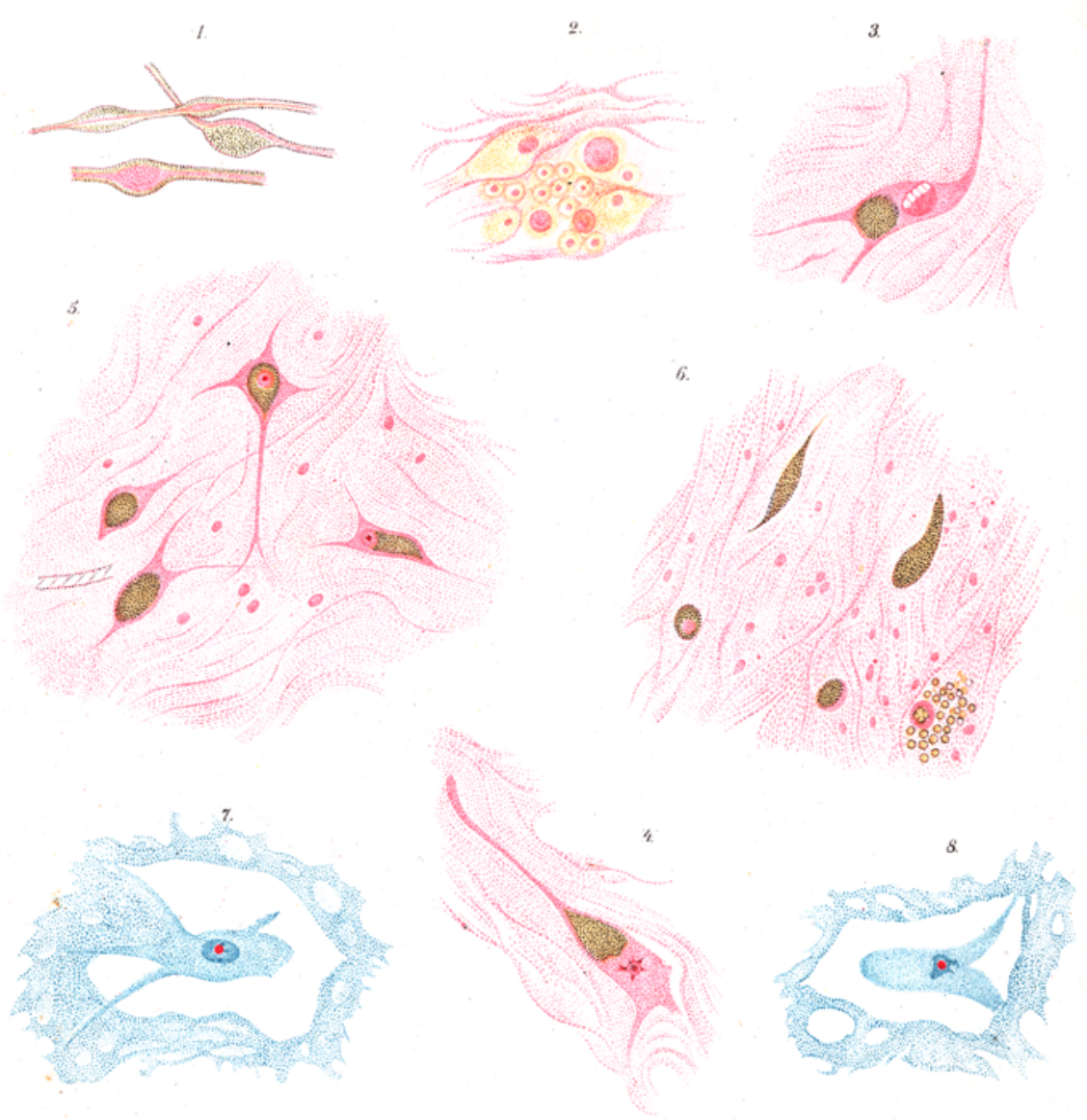

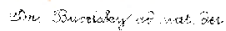


gänger nicht übereinstimmend, glaubt er ebenfalls, dass die hervorragendsten pathologischen Veränderungen bei der Hundswuth sich in den Gefässen des Centralnervensystems und in dessen juterstitiellem Gewebe befinden. Jedoch richtet Kolessnik off anch sein Augenmerk auf die Nervenelemente, in denen er eine Reihe pathologischer Prozesse bemerkt (zwar in einem sehr unansehnlichen Naassstabe). Er erwähnt, dass die Nervenzellen in allen Regionen des Gehirns und Rückenmarks von runden Zellenelementen umgeben sind, und, indem er einige isolirt hat, constatirt er in deren Protoplasma 2-4 Körperchen, welche den lymphatischen oder wandernden Zellen ähnlich sind. Weiter betont er, dass das Protoplasma der Nervenzellen sich gewöhnlich körnig und oft so trübe darstelle, dass man den Kern nicht deutlich sehen konnte.

Speciellere Nachrichten über die Veränderungen der Nervenzellen bei der Rabies finden wir bei Pokotiloff ${ }^{1}$ ). Er hat bei $z$ wei, in Folge von Hundswuth gestorbenen Personen das Centralnervensystem untersucht und fand in beidẹn Fällen, dass die Nervenzellen, was ihre Structur anbelangt, sich von den normalen beträchtlich unterschieden: sie hatten meistentheils undeutliche Contouren und manchmal waren anstatt der Nervenzellen nur kleine Häufchen von feinkörniger Substanz sichtbar. An den Stellen, wo die Zellen verhältnissmässig noch gut conservirt waren, erschienen sie vergrössert und ihr Protoplasma trübe und körnig; um den kaum bemerkbaren Kern Jag dagegen in Form eines Streifens eine kleinere oder grössere Menge von Pigment. Die Nervenfasern, wie Pokotiloff meint, zeigten fast niemals Veränderungen. Auf eigene Beobachtungen gestützt, nimmt der Verfasser an, dass bei der Rabies sich eine parenchymatöse Gehirnentzündung entwickele, welche jedoch nicht diffus, sondern in Form einzelner kleiner Heerde zerstrent sei. Am stärksten traten diese Nester in den Stirnlappen, in der Medulla oblongata und im Halstheile des Rückenmarks auf.

Diese Arbeit, nach welcher, wie wir es gesehen haben, die Nervenelemente in dem pathologischen Prozesse eine wichtige Rolle spielen, war lange Zeit die einzige. Die späteren Ver-

1) Patholog. Anatomie der Lyssa beim Menschen. Journal der Moskauer Chirurgischen Gesellschaft. 1875. Russisch. 
fasser kommen wieder auf die früheren Ansichten, welche ihre höchste Entwickelung in den obengenannten Untersuchungen von Benedikt und Kolessnik off erreicht haben, zurück. Wassilieff ${ }^{1}$ ) z. B. erwähnt nur oberflächlich die Betheiligung der Nervenzellen in dem Leiden, welches bei der Hundswuth das Gehirn und Rückenmark betrifft. Er sagt, dass einzelne Zellen der Medulla oblongata sich trübe, von undeutlichen Umrissen und mit kaum bemerkbarem Kerne zeigten. Aehnliche Veränderungen, aber bedeutend mehr sichtbare, fand er in den Purkinje'schen Zellen des Kleinhirns. Forel ${ }^{2}$ ) sah ausser Hyperämie und unbedeutender Stase der Lymphe unter der Adventitia keine anderen pathologischen Veränderungen im Centralnervensystem und betrachtete sogar die erwähnten als secundäre, durch Krampfanfälle hervorgerufen. Brigidi ${ }^{3}$ ) bemerkte Häufchen kugelförmiger, kleiner Körnchen in den Nervenzellen des Rückenmarks und der Medulla oblongata, sowie der Sehhügel. Schultze ${ }^{4}$ ) fand bei einem Hundswuth-Kranken die Nervenzellen im Centralnervensystem vollständig normal. Nirgends sah er varicöse Axencylinder und nur in den mit Blut überfüllten Gefässen, sowie im umgebenden Gewebe bemerkte er deutliche Körner von schwarzem Pigment. Er schliesst daraus, dass die Hundswath nicht zu den Krankheiten des Centralnervensystems, die zufolge einer Entzündung entstehen, gezählt werden könne.

Ohne die Reihe von Arbeiten überwiegend englischer Verfasser zu erwähnen, die die Mitwirkung der Nervenzellen im.pathologischen Prozesse bei der Lyssa entweder gar nicht annehmen oder nur flüchtig constatiren, sehe ich mich veranlasst; speciell die Untersuchungen aus den letzten Zeiten, welche diesen Gegenstand behandeln, zu beachten. Von diesen Erforschungen ist als eine der ausführlichsten die von $W$ eller ${ }^{5}$ ) zu erwähnen.

1) Ueber die Veränderungen des Gehirns und der Herzganglien bei der Lyssa. Centralb]. f. d. med. Wissensch. 1876.

$\left.{ }^{2}\right)$ Ueber die Hirnveränderungen bei Lyssa. Deutsche Zeitschrift für Thiermed. III.

3) Virchow's Jahresbericht. 1876.

4) Zur pathol. Anatomie der Chorea minor, des Tetanus und der Lyssa. Deutsches Archiv f. klin. Med. 1877, 20.

5) Ueber die Veränderungen des Gehirns und Rückenmarks bei Lyssà. Arch. f. Psychiatrie. 1879. 
Dieser Autor betrachtet, wie die meisten seiner Vorgänger, den pathologischen Prozess bei der Hundswuth als eine Entzündung, deren Ausgangspunkte das Gefässsystem und das. Bindegewebe sind. Diese Entzündung unterscheide sich jedoch von der gewöhnlichen Encephalitis oder Myelitis dadurch; dass die in ihrem Gefolge entstandenen Störungen in der Ernährung vicht besonders stark sind und keine Erweichung des durch den pathologischen Prozess ergriffenen Gewebes bedingen. Was die Nervenelemente anbelangt, so sagt. Weller, dass die Nervenzellen der Vorderhörner des Rückenmarks vollständig frei von deutlichen Fortsätzen waren, ihr Protoplasma oft verschwindende Umrisse zeigte und 2-3. Jymphatische Körperchen enthielt. Dessenungeachtet setzt er hinzu, dass seine. Untersuchungen überhaupt nur auf wenig zu beachtende Veränderungen in der Neuroglia und in den Nervenelementen fübrten. Indem der Verfasser die Erscheinungen im Gefässsystem alș solcho von grösster Bedeutung betrachtet, mạcht er die interessante Bemerkung, dass die Intensität der Veränderungen in den verschiedenen Abtheilungen des Centralnervensystems, sehr ungleich ist. Im Rückenmark betrafen sie fast ausnahmsweise nur die graue Substanz und besonders den den Centralkanal umgebenden Theil derselben; in der Medulla oblongata trat der Prozess am deutlichsten auch in der grauen Substanz, vornehmlich in der Nähe des IV. Ventrikels und der Raphe, besonders aber im Kerne der IX., X., XI. Nervenpare hervor. In den grauen Massen der Vierhügel, der Sehhügel und der gestreiften Körper waren die pathologischen, Veränderungen noch sehr deutlich, von da an aber nach den Frontallappen zu wurden sie immer schwächer und im Grosshirn verschwanden sie fast ganz... Solche Localisation fand. Weller bei Hunden; bei Menschen bemerkte er gewisse Aenderungen in derselben, und zwar waren in den Ganglia subcorticalia beinahe gar keine pathologischen Merkmale nachweisbar, gleichwie in den Kernen der III., IV., VII. und XII. Paare, und sonst im Grosshirn and Kleinhirn, d. h. beim Menschen ist der pathologische Prozess in."seiner Ausdehnung weit mehr beschränkt, indem er kaum die Medulla oblongata überschreitet, abèr er tritt in der Halsanschwellung des Rückenmarks bedeutend sichtbarer hervor, als beim Hunde. 
Auf Grund aller dieser Beobachtungen ist Weller zu dem Schlusse gekommen, dass bei der Lyssa sich eine acute Myelitis (Encephalitis) entwickele, welche übrigens wegen Mangel an Zeit nur auf das Anfangsstadium beschränkt bleibe.

Alle oben erwähnten Verfasser, mit Ausnahme von Pokotiloff vielleicht, haben jedoch nur wenig ihr Augenmerk auf die Veränderungen der Nervenelemente gerichtet, wiewohl sie manchmal diesen Veränderungen einen gewissen Werth zuerkannt haben. In der letzten Zeit erst hat Schaffer ${ }^{1}$ ) sich viel mit der Untersuchung der in den Nervenzellen und in den Nervenfasern des Rückenmarks bei den an Hundswuth gestorbenen Menschen vorkommenden Veränderungen beschäftigt. Für die am meisten charakteristische Thatsache sieht er das Erscheinen von Pigmentkörnein im Protoplasma der Nervenzellen an, welche Körner, gewöhnlich in kleinerer oder grösserer Anzahl an einem der Zellenpole sich ansammelnd, zaweilen solche Massen bilden, dass die ganze Zelle damit ausgefüllt wird. In diesem Falle bildete das normale Protoplasma gleichsam eine peripherische, Pigment enthaltende Scheide, in der weder der Kern noch das Kernkörperchen zu unterscheiden waren; vielmehr verschwanden dieselben und an jhrer Stelle traten Häufchen von Pigmentkörnern auf. Einige Zellen hatten noch Fortsätze, viele jedoch, dieser beraubt, stellten sich zusammengeschrumpft und abgerupdet dar. Manchmal traf man wieder ausschliesslich aus Pigmentkörnern bestehende Schollen, die ihrer Form nach den Nervenzellen entsprachen. Einzelne Zellen dagegen zeichneten sich durch besonders schwache Färbung des Protoplasma und vergrösserte Contouren aus; über den feineren Bau dieser Zellen kann jedoch nichts ausgesagt werden. Die pericellulären Räume fand Schaffer überall erweitert, was seiner Meinung nach durch das Gerinnen der Zellenkörper nach dem Tode allein sich nicht erklären lässt. Er ist der Ansicht, dass diese Erscheinung einen anderen Grund habe, und zwar, dass Exsudat, zwischen die Zelle und die sie umgebende Neuroglia tretend, auf beide einen Druck ausübe, was eine Störung in der Ernährung der Zellen nach sich ziehe und Vermehrung des Pigments zur Folge habe, welcher Druck An-

1) Histologische Untersuchung eines Falles von Lyssa. Arch. f. Psych. 1887. Bd. XIX. 
fangs auf Kosten des Protoplasma, später des Kernes und Kernkörperchens sich ausbilde. In ähnlicher ${ }^{*} W$ eise verändert erscheinen die Nervenfasern, besonders in den Vordertheilen der weissen Hinterstränge. Hier sah Schaffer anstatt der Nervenfasern von normalen Querschnitten oft nur runde, mit einer Scheibe umgebende Oeffnungen, die intensiv durch Carmin, aber durchaus nicht nach Weigert's Methode sich färbten; im Lumen einer solchen Scheibe befand sich gewöhnlich eine blasse körnige Substanz, die jedoch die Scheibe nicht ganz ausfüllte; an den Rändern vieler solcher Oeffnungen fand er nackte, zum Theil verdickte Axencylinder. Auf diese Thatsachen gestützt ist der Verfasser der Meinung, dass auch hier plasmatisches Exsudat zwischen die Nervenfasern und die sie umgebende Neuroglia eindringe und sich ein Druck auf die ersten entwickele.

Als Schluss seiner Untersuchung giebt Schaffer an, dass das Gift der Hundswuth im Rückenmark einen scharfen Entzündungsprozess hervorrufe, - eine acute Myelitis, welche das ganze Organ ergreife und am deutlichsten in der Halsabtheilung hervortrete. Eine solche Ausdehnung des Prozesses in Augenmerk nehmend ist er der Ansicht, dass die intensivsten Veränderungen in den Nervenelementen der Medulla oblongata sein müssen; diese hat er jedoch nicht untersucht, kann within auch seine Meinung nicht factisch bestätigen.

Die angeführten Resultate Schaffer's blieben nicht ohno Einfluss auf die späteren, diesem Gegenstande gewidmeten Arbeiten.

Vor einigen Monaten stand im Centralblatt fir Nervenbeilkunde ein Bericht des Prof. Laufenauer ${ }^{1}$ ) im Namen der ärztlichen Commission in Budapest, welche zur allseitigen Erforschung der Hundswuth eingesetzt war. Auf die Eigenthümlichkeiten des klinischen Bildes sich stützend, kamen die Mitglieder der Commission zu der Ueberzeugung, dass sie es mit einer acuten, im höchsten Grade infectiösen Krankheit zu thun hätten, deren eines Merkmal eine ausserordentliche Vergrösserung der Haut-, Muskel- und Sehnenreflexe, besonders in der Gegend der Bulbärnerven, sei. Die Schädlichkeit der Ansteckung sei um so stärker, je näher sich die Stelle der Verletzung am

1) Ueber Lyssa humana, 1889. No. 3. 
verlängerten Mark befinde; gleichzeitig damit werde auch die Incubationsperiode verkürzt. Die Ergebnisse der mikroskopischen Untersuchung brachten die Commission zu dem Schlusse, dass das Rückenmark bei der Hundswuth der Sitz einer diffusen Entzündung sei, welche sich sowohl auf die weisse wie graue Substanz verbreitet, und dass dieser Prozess auch die Nervenzellen nicht schone, welche einen hohen Grad pigmentöser Atrophie zeigen. Was die letztere anbelangt, so theilen die Aerzte von Budapest die Meinung Schaffer's, d. h. sie machen sie abhängig von dem Drucke, welchen das Entzündungsinfiltrat, das die pericellulären Räume reichlich ausfüllt, ausübt. Auf diese Weise erklären sie auch die Veränderungen in der weissen Substanz des Rückenmarks, wo, obgleich der Prozess viel schwächer hervortritt, derselbe doch deutlich, besonders in den Hintersträngen, markirt sei: die Myelinscheiden stellen sich hier hypertrophisch, zerfallend und stellenweise geronnen dar; das auseinanderfallende Myelin tritt meistentheils in Form von Tropfen nach aussen heraus.

Nach der Aufzählung der durch die Commission erlangten Resultate sagt Prof. La ufenauer: "diese Resultate unterscheiden sich von den durch andere Verfasser erzielten dadurch, dass sie bedeutende Veränderungen der Nervenzellen und Fasern zeigen. Benedikt, Kolessnik off, Forel, Gowers, Coats und Weller haben die perivasculären Entzündungsheerde und Blutaustritte beschrieben, die Nervenelemente fanden sie dagegen ganz verschont. Den Veränderungen der letzteren schreiben wir gegenwärtig desto grössere Geltung zu, da wir mit Leichtigkeit wichtige Symptome eben durch dieselben erklären können."

Mit diesen Worten Laufenauer's ist es schwierig vollständig einverstanden zu sein. In der That haben wir oben gesehen, dass auch früher viele Verfasser ihr Augenmerk auf die Veränderungen der Nervenelemente bei der Hundswuth gerichtet haben, einer von ihnen (Pokotiloff) denselben sogar eine sehr wichtige Rolle zuerkannt hat. Alle diese Forscher jedoch haben diese Veränderungen jeder etwas anders aufgefasst; sie waren geneigt, in denselben Zeichen einer Betheiligung der Nervenelemente zu sehen. Dagegen spielen nach Schaffer und Laufenauer diese Elemente eine ganz passive Rolle.

Die Aufklärung dieses Widerspruches, eine specielle Dar- 
stellung der Veränderungen der Nervenelemente im Centralnervensystem, ist das Ziel der vorliegenden Untersuchung.

Martin Parusew, ein Landmann, trat am 17. Juli 1889 in das Hospital des heiligen Johannes a Deo mit kurzen anamnestischen Nachrichten ein, aus denen ersichtlich war, dass er am 20. Mai von einem tollen Hunde in den linken Vorderarm gebissen wurde; vor einigen Tagen war er nach Warsebąu gekommen, um nach Pasteur's Methode eine Cur zu unternehmen, musste jedoch in Folge einer hochgradigen Aufregung in's Hospital gebracht werden.

Der Krauke, etwa 40 Jahre, alt, von regelmässigem, starkem Bau, befindet sich fortwährend in der höchsten Aufregung, spricht sinnlos, schreit, singt, wirft sich auf die Umgebenden, will aus dem Zimmer fliehen, antwortet auf die gestellten Fragen nicht. Bei jedem Versuch, dass er etwas trinke, treten sofort charakteristische Krämpfe ein. Die physische Untersuchung konnte wegen starker Aufregung nicht vorgenommen werden.

18. Juni. In der Nacht hat er nicht geschlafen; am Tage wie vorhin unruhig; er zerreisst die Kleider, wirft sich auf die Menschen und kann weder essen noch trinken.

19. Juni. Der Zustand ist unverändert. Der Kranke wirft sich auf die Leute und hat den Diener gebissen.

20. Juni. Erbrecben von Galle, nacb welchem ein schnelles Abnehmen der Kräfte eintrat.

In der Nacht ist der Kranke gestorben.

Die 12 Stunden nach dem Tode erfolgte Section ergab Folgendes:

Die Leiche bedeutend. abgemagert. Auf der Haut des linken Vorderarms ist ein Mal vom Bisse sichtbar. Das Fettgewebe ist schwach entwickelt. Die Muskeln auf dem Durchschnitt von duntelrother Farbe. Der Schädel hat regelrechte Formen; die Knochen des Schädels dünn; Diploë mässig entwickelt. Dura leicht vom Knochen zu trennen; ihre äussere Fläche bedeutend byperämisch, die innere glänzend und glatt. Pia hyperämisch, in der Gegend der Rolando'schen Furche etwas trübe, lässt sich aber leicht von der ganzen Oberfäche des Gehirns abnehmen. Die Gefässe an der Gehirnbasis und in den Sylvi'schen Spalten mit Blut überfüllt, ibre Wände unverändert. Die Gehirnsubstanz hart, stark geröthet (besonders die wieisse Substanz und die Corpora caudata; die beiden Sehhügel bedeutend weniger). Eine starke Hyperämie ist im Pons Varolii und im Ǩleinhirn sichtbar. Die Häute des Rückenmarks und seine Substanz sehr hyperämisch. Die rechte Lunge liegt bequem im Brustkasten, ist durch und durch stark geröthet, entbält überall Luft. Die linke Lunge jst an die Wände des Brustkastens angewachsen, im Durehsehnitt rosenroth, trocken, enthält überall Luft; der Rand des unteren Lappens emphysematisch. Das Herz im Querschnitt vergrössert; der rechte Ventrikel mit dicker Schicht Fett belegt, die Wände des linken bedeutend rerdickt. Die Milz sehr klein, ihre Kapsel geschrumpft, die Substanz von dunkler Zimmetfarbe, ziemlich hart, die Trabeculae dent- 
lieh sichtbar. Die Leber von gewöhnlicher Grösse, ihr Gewebe im Durchschnitt stark blutreich. Die Nierenkapsel dünn, leicht abnehmbar, die Oberfläcbe der Nieren glatt und glänzend, die Rindensubstanz blass; die Pyramiden stark blutreich. Der Tractus ventriculo-intestinalis zeigt nichts Anortales.

Ein Stück des Rückenmarks und ebenso eines aus den Hirnganglien wurde gleich nach der Obduction in Sublimatlösung gelegt; das übrige Centralnervensystem, gütigst in toto durch den Chefarzt Dr. A. v. Rothe zu meiner Untersuchung gestellt, blieb in einer Auflösung von Kalium bichromicum 6 Monate lang bis zur völligen Härtung.

Mikroskopische Untersuch u ng.

Bei der mikroskopischen Untersuchung fallen zunächst die Veränderungen des Gefässsystems in die Augen; die Erörterung des Charakters und der Eigenthümlichkeiten dieser Veränderungen gehört jedoch nicht in den $\mathrm{Be}$ reich gegenwärtiger Arbeit. Ich beschränke mich daber nur zu erwähnen, dass dieselben mit den Beschreibungen obengenannter Autoren im Allgemeinen übereinstimmen; ebenso, wie die früheren Forscher, konnte ich sehr zahlreiche Blutaustritte, Ueberfüllung der Gefässe mit Blutkörperchen, Massen plasmatischer Exsudate und emigrativer Elemente um die Gefässe, dichte Infiltration der Gefässwände mit Kernen und diffuse Zelleninfiltration der weissen sowohl, wie der grauen Substanz constatiren. Solche Veränderungen wurden überall im ganzen Centralnervensystem angetroffen, aber in einzelnen Stellen erreichten sie die höchste Entwickelung besonders im verlängerten Marke, theilweise auch in Hirnganglien. Was die Nervenelemente anbelangt, auf welche ich hauptsächlich das Augenmerk gerichtet babe, so waren die Veränderungen derselben in versehiedenen Regionen des Nervensystems, wiewohl sie einen und denselben Charakter hatten, in ganz verschiedenem Maassstabe entwickelt; daher erfordern sie eine specielle Beschreibung.

I. Das Rückenmark.

a) Die weisse Substanz ${ }^{1}$.

In Längsschnitten verschiedener Abtheilungen des Rückenmarks sieht man zuerst, dašs an der Mehrzahl der Nervenfasern die Myelinscheide eine

1) Die ganze bier angeführte Beschreibung ist auf Grund der Untersuchung von Präparaten, welche nach Härtung in einer Auflösung von Kali bichromicum erbalten worden, zusammengestellt, wobei anfangs 1 procentige, später $1 \frac{1}{2}$ procentige und endlich 2 procentige Lösung von mir angewendet wurde. Einige Stücke des Centrainervensystems waren jedoch durch den pathologischen Prozess dermaassen erweicht, dass ich, um die genügende Härte zu gewinnen, dieselben aus dem Kal. bichr. in Alkohol zu übertragen genöthigt war, in welchem sie einige Tage liegen mussten, bevor sie zur Vorbereitung solcher Schnitte, in weichen man die topographische Lage der pathologischen Veränderungen bezeichnen konnte, tauglich waren. Die Schnitte wurden mit Am- 
Reibe von rosenkranzartigen Verdickungen bildet, demzufolge das mikroskopische Bild ein sehr sonderbares Aussehen hat. Wenn man diese varicöse Verdickungen genauer beobachtet, so ist es leicht zu erkennen, dass der Axencylinder daran gar keinen Antheil hat, sondern gewöhnlich nach der Peripherie gerückt ist. In einigen Fasern bemerkt man jedoch, dass er, entsprechend der Verdickung der Markscheide, gleichfalls spindelartig angescbwollen ist. Verhältnissmässig selten findet man so bedeutende Anschwellungen der Axencylinder, dass die rosenkranzartigen Verdickungen der Nervenfasern fast ausschliesslich von ihnen abhängen; in solchen Fällen . zeigt sich die Myelinschicht sogar verdünnt (Fig. 1). An Querschnitten kann man seben, dass fast alle Nervenfasern wit einer Myelinscheide, welche jedoch in der grössten $\mathrm{Zahl}$ der Fasern bedeutend verdickt ist, bedeckt sind; überall aber bebält sie das normale Verbältniss zur Carminfärbung, sowie ihre gewöhnlichen optischen Eigenschaften; die Axencylinder stellen sich in verschiedenem Grade hypertropbisch dar, und einige derselben liegen, frei von Myelin, direct und fest an der sie umgebenden Neuroglia an (Fig. 2). Solche Veränderungen findet man ebenso in den weissen Seiten-, wie in den Hinter- und Vordersträngen, so dass es schwer zu entscheiden ist, wo sie den höchsten Grad der Entwickelung erreichen.

b) Graue Substanz.

Die pericellulären Räume stellen sich sehr undeutlich dar. Die Nervenzellen stossen dicht an das umgebende Gewebe, haben mehr oder weniger gerundete Formen und nicht selten entbehren sie gänzlich der Fortsätze. Im Protoplasma der Nervenzellen befindet sich (mit wenigen Ausnahmen) eine bedeutende Anzahl kleiner, gelblichbrauner Pigmentkörner, welche sich grösstentieils entweder um den Kern, oder an einem der Zellenpole sammeln. Ziemlich oft trifft man Zellen an, in denen die Pigmentkörner den ganzen Leib einnehmen, indem sie nur die peripherische Schicht frei lassen; in solchen Zellen kann man weder den Kern noch das Kernbörperchen unterscheiden (Fig. 5), während man, in anderen, wie es scheint, den unveränderten Zellenkern seben kann, obwohl der ganze Zellenkörper gleichsam aus Pigmentkörnern zusammengesetzt ist.

Solche Veränderungen der Nervenzellen treten überbaupt deutlicher in den Vorder- als in den Hinterbörnern bervor und werden in der ganzen Ausdebnung der Lenden- und Brustabtheilung des Rückenmarks vorgefunden, sind aber vielleicht in der ersteren mehr, als in der letzteren, sichtbar. Was den Halstheil anbelangt, so haben hier die Zellenveränderungen, wiewohl sie denselben Charakter beibehalten, eine bei Weitem grössere Intensität. In den Gruppen der grossen Zellen der Vorderbörner, beşonders in der Nähe der Gefässe, trifft man sehr oft Nervenzellen obne Fortsätze, eiförmig und

moniumcarmin oder Pikrocarmin Hoyer's gefärbt und theils in Glycerin, theils vorber mit Alkohol entwässert und mittelst Nelkenöl durchsichtig gernacht, in Canadabalsam mikroskopisch untersucht. 
so mit Pigment überfüllt, dass man kaum bier und da regelrecht gefärbtes Protoplasma erblicken kann. Unter den einzelnen Zellengruppen ist am meisten die centrale, welche in der Gegend des Centralkanals liegt, verändert. In der eben erwähnten Abtheilung des Rückenmarks kann man auch Veränderungen in den Kernen der Nervenzellen bemerken, Veränderungen, die, wiewobl sie auch in den vorherigen vorkommen, doch in viel geringerem Grade erscheinen. Die Kerne haben hier oft unregelmässige Formen, sie sind eckig, pyramidenartig verlängert; einige von ibnen sind au den Rändern wie zersetzt oder entbalten in sich rundliche kleine Vacuolen (Fig. 3 u. 4). Die Kernkörperchen zeigen keine sichtbaren Veränderungen. Nicht zu selten sieht man veränderte Zellenkerne, welehe in einem, ein verhältnissmässig unansebnliches Quantum von Pigment besitzenden Protoplasma sich befinden, und umgekehrt sieht man oft augenscheinlich sehr gut conservirte Zellenkerne in einem Protoplasma, das mit Pigment überfüllt ist (Fig. 6).

\section{Medulla oblongata und Pons Varolii ${ }^{1}$ ).}

Die Veränderungen der Nervenzellen erreichen bier eine bei weitem grössere Intensität, als in der vorhergebenden Abtheilung. Sie betreffen sowobl den Zellenkörper, als den Zellenkern. Der Zellenkörper ist mit Pigment überfüllt; der Kern befindet sich gewöhnlich an einem der Zellenpole, unterscheidet sich durch intensive Carminfärbung und ist oft von einer zersetzten oder verschrumpten Form sogar in denjenigen Zellen, welche eine verhältnissmässig unbedeutende Menge von Pigment besitzen. Wiewohl die Veränderungen der Nervenzellen im Allgemeinen denselben Charakter beibehalten, so ist es namentlich diese Abtheilung des Nervensystems, in welcher dieselben, wie ungleich sie auch in den verschiedenen Gruppen ausgedrückt sind, leicht $z u$ bemerken sind. Am stärksten treten sie in den Kernen des XII. Nervenpaares bervor, wo die Zahl der Zellen bedeutend kleiner ist; von den übrigen aber werden solche vorgefunden, deren ganzer Körper mit Pigment äberfüllt ist. Manchmal lassen sich in denselben zwischen den Pigmentkörnern noch Ueberreste von regelrecht gefärbtem Protoplasma bemerken; andere Zellen dagegen stellen sich nur als Pigmentschollen dar, welche durch Gestalt und Lage an Zellenelemente erinnern (Fig. 6).

Neben so veränderten Zellen trifft man nicht selten kleine Häufchen von Pigmentkörnern von regellosen Contouren an, über deren Herkunft sich nichts Bestimmtes sagen lässt. Am meisten verschont sind die Zellen der Substantia gelatinosa und der Oliven; in diesen Regionen unterscheiden sich die Nervenzellen einzig und allein ron den normalen durch ein grösseres

1) Die Substanz des verlängerten Markes erlangte am schwierigsten eine vollständige Härtung in Kali bichr., daher musste man dieselbe auf einige Zeit in Spiritus legen (anfangs in 50, später in 90procentigen). In den, der Wirkung des Spiritus ausgesetzten Präparaten waren die pericellulären Räume etwas mehr erweitert, als in denen, welche nur in der Kalilösung gehärtet worden. 
Quantum von Pigment im Protoplasma, ihre Kerne dagegen haben deutliche regelmässige Contouren und eine runde, manchmal etwas verlängerte Form.

Sehr bedeutende Veränderungen finden wir in den Zellen, welche zu. den Kernen der XI., $\mathbf{X}$. und IX. Nervenpaare gebören, wo ebenfalls verbältnissmässig oft nur Pigmentschollen geseben werden. In den mebr nach vorn gerücteten Abtheilungen der Kerne des IX. Paares werden diese Veränderungen sichtlich schwächer, aber auch hier treten sie ohne Vergleich mehr, als in der Gegend des Kernes des VIIL. Paares, bervor. Im Kerne der Acustici giebt es keine vollständig mit Pigment ausgefüllte Zellen mebr; im grössten Theile dieses grossen Territoriums finden wir die Nervenzellen vollständig gut conservirt: sie unterseheiden sich von den normalen einzig und allein durch ein grösseres Quantum des das Protoplasma ausfüllenden Pigments. Die einzelnen grossen Zellen, welche an beiden Seiten der Raphe liegen, sind in ganzer Länge des verlängerten Marks sebr beschädigt. Die Zellen des Funiculus teres und des Pons sind kaum verändert und unterscheiden sich in dieser Hinsicht nicht von denen der Oliven. Die Zellen der Kerne des VI. Nervenpaàres sind sehr verändert, wiewobl unter denselben schon keine vollständig mit Pigment erfüllten angetroffen werden. Die sensorischen Kerne des V. Nervenpaares sind verbältnissmässig wenig verändert; sie unterscheiden sich nur durch Anschwellung, Fortsatzmangel, Trübung des Protoplasma, in welchem sich ein unbedeutendes Pigmentquantum befindet. Die Zellen der motoriscben Kerne des V. Nervenpaạres stellen sich im höheren Grade verändert dar. Die Zellen in den Keraen des IV. Nervenpaares sind iw höchsten Grade verändert, ihre Körper meistentheils vollständig mit Pigment erfüllt, obgleich die Zellenkerne und Kernkörperchen zu erkennen sind. Besonders deutliche Veränderungen zeigen die Solitärzellen, die nach innen vom Kerne des N. trochlearis liegen: unter denselben findet man kaum solche, in welchen der Kern durch das Pigment sichtbar wäre ${ }^{1}$ ).

\section{Die Hirnganglien:}

In den Kernen der vorderen Vierhïgel enthalten die Nervenzellen sebr viel Pigment, haben jedoch ibre gewöbnliche Form. Bei weitem deutlicher treten die Veränderungen in den grauen Kernen der binteren Vierbügel hervor, wo oft nur Häufchen von Pigmentbörnern angetroffon werden, welche durch ibre Contouren lediglich an Zellen mit Fortsätzen erinnern; manchmal sieht man in solchen Häufchen den Kern, welcher entweder wenig verändert ist, oder eine eckiga unregelmässige Form, als wenn er zusammengeschrumptt wäre, besitzt. Im Sebhügel sind die zum Pulvinar gehörenden Zellen wenig verändert: der Kern und das Kernkörperchen deutlich erkennbar, das Protoplasma entbält ein nur unbedeutendes Quantum Pigment. Die Zellen in den vorderen Theilen der Sehbugel haben meistentheils Kerne von unregelmässigor Form, Fänglich, oval, pyramidenförmig u.s.w.

1) Das Gebiet des Kernes der N. oculomotorii konnte ich wegen Bescbädigung bei der Section nicht untersuchen. 
In allen Zellenleibern befindet sich eine grosse Menge von Pigment und nicht selten trifft man Zellen an, die nur aus ovalen, dureb Carmin dunkel gefärbten Kernen bestehen, welche von Pigmentkörnern umgeben sind. Nebenbei findet man oft unregelmässige Gruppen von Pigmentkörnern obne Kern. Corpus caudatum: Die Nervenzellen, mit grossen runden Kernen und einem geringem Quantum von feinkörnigem Protoplasma, enthalten nur unbedeutende Pigmentmassen von demselben Charakter.

\section{Das Kleinhirn.}

In den Purkinje'schen Zellen befindet sich eine ziemlich grosse Menge von Pigmont, dieses aber hindert nicht, den Kern und das Kernkörperchen unterscheiden zu können. Die Form des Zellenleibes unverändert, die Fortsätze gut erhaiten. Die übrigen Nervenelemente der Rinde zeigen keine sichtbaren anomalen Erscheinungen. Die Zellen in den Centralkernen des Kleinhirns haben ibre gewöhnliche rundliche Gestalt und enthalten so reichliche Pigmentanbäufungen, dass der Kern nicht deutlich zu erkennen ist.

\section{Die Grosshirarinde.}

a) Stirnlappen.

Die Veränderungen erreichen den höchsten Grad in den grossen und kleinen Pyramiden. Die pericellulären Räume sind erweitert und enthaiten oft emigrative Elemente. Solche Erweiterungen findet man fast nur an der Zellenbasis, seltener erstrecken sie sich über den ganzen pericellulären Raum. In allen Pyramidaliellen ohne Ausnahme sind ansehnliche Anhäufungen von Pigmentkörnern zu seheu; diese Körner, gewöhnlich an der Zellenbasis angesammelt, machen den Eindruck, als wenn sie den Kern nach der Peripherie drängten; in anderen Zellen dagegen zerstreuen sie sich und umgeben von allen Seiten den Kern, der oft ungleiche, eckige oder pyramidenförmige Contouren hat, aber sehr deutlicb bemerkbar ist. Im Protoplasma der Zellen sind oft kleine, leere Stellen von runder Form (Vacuolen) oder emigrative Elemente. Nicht zu selten kommen Zellen vor, deren ganzes Protoplasma. vollständig mit Pigment ausgefüllt ist, jedoch ist der Kern, wenn er noch sichtbar ist, intensiv durch Carmin gefärbt und behält seine gewöbnliche Form; sebr selten sind in den pericellulären Räumen unförmliche Gruppen von Pigmentkörnern sichtbar, die den unveränderten Zellenkern umgeben. Was die kleinen abgerundeten Nervenzellen anbelangt, so haben die Veränderungen in denselben einen etwas anderen Charakter, bauptsächlicb das Protoplasma betreffend: ịhre Kerne sind gewöhnlich gut erhalten, aber meistentheils von einem unbedeutenden Quantum feinkörniger Substanz umgeben, in der man nur selten einzelne Körner des oben erwähnten Pigments bemerken kann; in einigen pericellulären Räumen sieht man einzig den frei liegenden Kern.

b) Paracentrallappen.

Die Veränderungen der Nervenzellea, welche denselben Charakter, wie in der vorhergebenden Abtheilung, beibehalten, sind sehr genau in's Auge 
fallend. In den Riesenzellen kann man ausser reichlichen Pigmentmassen im Protoplasma oft unregelmässige Kerne, bemerken, welche geschruiopft erscheinen.

c) Hinterlappen.

Die Veränderungen in den kleinen Nervenzellen haben denselben Cbarakter, wie in den anderen Theilen; die Pyramidenzellen enthalten nur eine unbedeutende Masse von Pigment.

Ein Theil der Halsanschwellung des Rüekenmarks, wie auch kleine Stücke der Sebbügel, der Stimlappen und Paracentrallappen der Gehimrinde wurden, wie schon oben erwähnt, sofort nach der Section in gesättigte Sublimatlösung gelegt. Die aus den anf diese Weise gebärteten Präparaten erbaltenen Schnitte wurden nach Gaule's Methode gefärbt (mit Hämatoxylin, Nigrosin, Eosin und Safranin) und in Canadabalsam untersucht. In diesen Schnitten fällt zunächst die ungebeure Vergrösserung der pericellulüren Räume in's Auge: die Zellen liegen in denselben ganz frei und werden nur mittelst der Fortsätze mit dem umfassenden Gewebe verbunden. Im gleichmässig bläulich gefärbten Protoplaswa siebt man meistentheils sehr deutlich den Kern von derselben Farbe und scbarfen Contouren; in allen Zellen obne Ausnahme enthält er ein rundes dunkel-himbeerfarbiges Kernkörperchen und eine Unmasse kleiner dunkelblauer, ziemlich gleichmässig über den ganzen Kern zerstreuter Körner. Unter den letzteren finden wir gröbere Körner von derselben Farbe, die in unregelmåssigea Gruppen sich um das Kernzörperchen ansammeln (Fig. 7). Die Zahl solcher Körner ist sogar in einem und demselben Schnitte in verschiedenen Zellen sehr verschielen: in den einen sieht man nur einzelne Körner, in den ancieren bilden sie eine feste, fast den ganzen Kern umfassende Masse. In noch anderen Zellen zeichnen sich die Kerne durch unregelmässige, gezackte, nicht immer deutliche Contouren aus. Endlich trifft man auch solche Zellen an, in denen man anstatt des Kernes nur dunkel-himbeerfarbige Kernkörperchen sieht, die von einer gewissen Anzabl grober Körner von oben erwähntem Charakter umgeben sind (Fig. 8). Die auf diese Art veränderten Kerne gebören oft $z u$ Zellen, deren Protoplasma verbältnissmässig wenig Pigment enthält, und umgekehrt ist es nicht schwer, eine Zelle mit einem Protoplasma, welches mit Pigment überfüllt ist, und mit einem gut conservirten Kerne zu finden. Besonders oft kann man bedeutende Veränderungen im Bau der Kerne in den Zellen der Vorderhörner des Rückenmarks und in den grossen Zellen der Paracentrallappen bemerken.

Bei der Analyse und Beurtheilung der Resultate der mikroskopischen Untersuchung werfen sich $z$ wei wichtige Fragen auf: die erste betrifft den Charakter der von uns beobachteten Veränderungen, die andere die ungleiche Verbreitung derselben, ihre Localisation im Centralnervensystem.

Wir haben gesehen, dass in unserem Falle die Nerven- 
fasern, wie auch die Nervenzellen deutliche Veränderungen darbieten.

An den Nervenfasern fallen zuerst auf die rosenkranzartigen Verdickungen der Myelinscheide. Die Veränderungen der Contouren der die Axencylinder amgebenden Myelinschicht können bekanntlich, wenn die chemischen Eigenschaften der letzteren sich nicht gleichzeitig ändern, für unbediagt pathologische Erscheinung nicht angesehen werden. Nach Pertig ${ }^{1}$ ) haben dann auch andere Autoren dargethan, wie schnell und stark eine Myelinschicht ihre Formen sogar unter Einfluss gleichgültiger Reagentien ändern kann. Der Vergleich jedoch der Schnitte aus dem Gehirn unseres Wuthkranken mit den stereotyp auf dieselbe Weise von einem Gesunden erzielten, dann die Verbreitung der Verdickungen, die an dem grössten Theile der Fasern bemerkbar sind, endlich die fast mathematische Gleichförmigkeit dieser Anschwellungen, -- alles das berechtigt uns, dass wir darin einen offenbaren, in der weissen Substanz des Centralnervensystems entwickelten pathologischen Prozess sehen.

Diese Ansicht wird durch das Verhalten der Axencylinder bestätigt. Wie schon oben erwähnt ist, umgeben die Axencylinder, welche ihre Gestalt nicht verändern, einige Myelinverdickungen, an der Formirung anderer dagegen nehmen sie mehr oder weniger thätigen Antheil. In einzelnen Fällen konnte man sich sogar überzeugen, dass diese Anschwellungen der Axencylioder riesenhafte Dimensionen erreichen und fast die ganzen Verdickungen der Nervenfasern ausfüllen, von einer dünnen Myelinschicht kaum bedeckt. Endlich gab die Beobachtung der Querschnitte, ausser dass sie die in den Längsschnitten bemerkten Thatsachen zu sehen erlaubten, noch die Möglichkeit, das Vorhandensein verdickter Axencylinder, welche Myelin ganz entbehrten, zu constatiren.

Hier wirft sich aber eine Frage, welche bis jetzt noch nicht bestimmt gelöst worden ist, auf, nehmlich die nach der Bedeutung der rosenkranzartigen Anschwellungen des Axencylinders.

Wiewohl (harcot ${ }^{2}$ ), Joffroys), Hayem ${ }^{4}$ ), Westphal ${ }^{5}$ )

1) Archiv für mikroskopische Anatomie. 1881. H. 2.

5) Leçons ete. 1874-1877.

3) Comptes rendus des séances et mémoires de la société de biologie. 1873 .

4) Archives de physiologie normale et pathol. 1874. V.1.

5) Westphal's Archiv Br. IX. S. 243 ff. 
und viele andere bestimmt diese Verdickungen für das Zeichen eines thätigen Entzündungsprozesses ansehen wollen, hat sie Obermeier ${ }^{1}$ ) auch im normalen Gehirn gefunden, freilich mit dem Vorbehalt, dass sie in diesem Falle nicht grosse Dimensionen erreichen.

Unter den neuesten Verfassern hält Obersteiner ${ }^{2}$ ) sie geradezu für ein Zeichen des Entzündungszustandes. Friedmann ${ }^{3}$ ) kam aber auf Grund zahlreicher Beobachtungen zu dem Schlusse, dass man das Vorhandensein activer Prozesse in den Axencylindern überhaupt für nicht erwiesen ansehen kann; im einzelnen dagegen lenkt dieser Forscher, indem er zur Aufklärung der Bedeutung der angeschwollenen Axencylinder übergeht, das Augenmerk darauf, dass sie, besonders im Rückenmark, schon dann, wenn noch keine Spur von irgend welchen anderen Veränderungen in den Nervenelementen und im Bindegewebe vorhanden ist, erscheinen. Danach können einzelne hypertrophische Axencylinder nicht bestimmt eine parenchymatöse Entzündung darthun, sondern nur das Anfangsstadium derselben andeuten; andererseits erreichen solche Hypertrophien oft colossale Dimensionen und zwar in sehr kurzem Zeitraum, sogar in einigen Stunden. Wunderlich wäre die Annahme, dass die Zellenelemente nicht zuerst einen energischen Antheil an dem pathologischen Prozesse haben sollten. Man könnte eher meinen, dass wir es hier nur mit einer passiven Anschwellang zu thun haben.

Ohne Rücksicht darauf, dass diese Meinung sehr wahrscheinlich ist, darf man nicht doch ausser Acht lassen, dass sie nur auf einer Annahme a priori basirt. Ausserdem verwirft auch Friedmann den pathologischen Charakter der Hypertrophie des Axencylinders nicht, er zweifelt nur an ihrer activen Entstehung.

Trotz dieser ganz entgegengesetzten Ansichten kormmen fast alle Verfasser im Allgemeinen zu der Uebereinstimmung, dass die Hypertrophie des Axencylinders eine pathologische Erscheinung ist. Eine Ausnahme hiervon scheint nur Obermeier zu machen. Er begeht denselben Fehler, wie R. Schultz ${ }^{4}$ ), welcher,

1) Dieses Archiv. 1873.

2) Handbuch zur Lehre über den Bau des Centralnervensystems. 1886.

3) Archiv f. Psych. Bd. XIX.

4) Neurologiscbes Centralblatt. 1883. No. 23 . 
nachdem er in den Zellen des Rückenmarks gesunder Menschen Vacuolen gefunden hatte, solchen Erscheinungen den pathologischen Charakter abstreitet. Ebenso, wie einzelne vacuolisirte Zellen im Gehirn eines gesunden Menschen angetroffen werden können, beweisen einzelne hypertrophische Axencylinder das Vorhandensein eines krankhaften Prozesses nicht; wenn aber diese Erscheinung an dem grösseren Theile der Nervenfasern existirt, so versteht es sich von selbst, dass dieselbe eine ganz andere Bedeutung hat. In unserem Falle sind die Veränderungen fast an allen Fasern der weissen Rückenmarkstränge vorgekommen und sie müssen ohne Zweifel als pathologisch angesehen werden.

Ohne eine genauere Erklärung über ihren Charakter geben za wollen, - um so mehr, da bis jetzt die Frage über die Mitwirkung der Nervenzellen in dem Entzündungsprozesse sehr mangelhaft bearbeitet worden ist, - füge ich nur hinzu, dass, soviel aus meinen Schnitten zu schliessen ist, der pathologische Prozess im Myelin, welches rosenkranzartig anschwillt, beginnt; die Axencylinder, welche anfangs an der Erzeugung dieser Anschwellungen keinen Antheil nehmen, werden allmählich in diesen Prozess hineingezogen, sie hypertrophiren in entsprechenden Segmenten und füllen nach und nach die ganze Verdickung, indem sie das Myelin verdrängen. Endlich verschwindet die Myelinschicht und der hypertrophische Axencylinder bleibt entblösst. Was mit ihm später geschieht, davon konnte ich mich nicht überzeugen.

Was nun die Nervenfasern im Gehirn anbelangt, so sind hier die Veränderungen dieselben, wie im Rückenmark, aber bedeutend weniger deutlich. In den Nervenzellen des Centralnervensystems haben wir überall nur eine Art von Veränderungen gesehen, die sich durch ihre Intensität unterschieden. Sie betrafen sowohl das Protoplasma, als auch den Kern. Im Protoplasma haben wir stets gelbgraues körniges Pigment angetroffen, welches entweder an einem der Zellenpole lag, oder den Kern umgebend die peripherische Schicht des Protoplasma frei liess. In den von dem Prozesse am meisten betroffenen und mit Pigmentkörnern angefüllten Zellen, welche in solchen Fällen eine rundliche Form annahmen und ihrer Fortsätze beraubt waren, konnte man weder den Kern, noch das Kernkörperchen mehr 
erkennen. Neben ähnlichen Zellen befanden sich Eäufchen von Pigmentkörnern, welche nur durch ihre Gestalt an Nervenzellen erinnerten; manchmal war in ihnen der Kern erkenubar. Aus der Zusammenstellung dieser Erscheinungen ist es sichtbar, dass wir es mit pigmentöser Atrophie, welche die Nervenzellen zum vollständigen Verschwinden führt, zu thun haben.

Eben solche Veränderungen des Protoplasma hat, wie schon oben gesagt, auch Schaffer beschrieben. Dieser Autor erklärt sie aber als Folge des Druckes der Zellenkörper durch das Entzündungsinfiltrat, welches die pericellulären Räume ausfüllt. Meine Präparate erlauben durchaus nicht die Annahme einer solchen Erklärung.

Wir haben gesehen, dass die pericellulären Räume nach der Erhärtung im Kal. bichrom. nicht sehr bedeutend und jedenfalls nicht grösser als im normalen Gehirn waren. Dagegen erreichten sie bei der Erhärtung im Sublimat colossale Dimensionen, aber dieselbe Erscheinung habe ich unter denselben Verhältnissen im normalen Gehirn bemerkt. Die Grösse der pericellulären Räume hing daher lediglich von der Art und Weise ab, wie die Präparate vorbereitet wurden. Deshalb ist kein Grund vorhanden, die grössere Entwickelung des Pigments von dem durch das Entzündungsexsudat auf die Nervenzellen ausgeübten Dracke abhängig zu machen. Es ist daher durchaus anzunehmen, dass das Pjgment im Protoplasma vermöge einer selbständigen Störung ihrer Ernährung, welche erstere durch von uns unbekannte Bedingungen bervorgerufen ist, ausgebildet wird. Und in der That treffen wir sehr oft in der pathologischen Anatomie des Nervensystems sogenannte pigmentöse Atrophie an, - eine Erscheinung, über deren Bedeutung man bis jetzt noch nicht genügend einverstanden ist.

Von einer solchen Atrophie sprechen Arnd ${ }^{1}$ ), Frommann ${ }^{2}$ ), Charcot ${ }^{3}$ ) u. A. Charcot erwähnt, indem er stufenweise die progressive Entwickelung des atrophischen Prozesses in den Nervenzellen beschreibt, dass er diesen oft bei den, anfänglich in

1) Dieses Archiv. 1874. Bd. 59.

2) Ueber die Gewebsveränderungen bei der multiplen Sklerose des Gehirns und Rückenmarks. Jena 1878.

3) Leçons etc. T. II. p. 185. 
der benachbarten Neuroglia sich entwickelnden Eutzündungen und bei verschiedenen chronischen Leiden des Centralnervensystems (wie z. B. bei der Bulbärparalyse, progressiven Muskelatrophie u. s. w.) vorgefunden hat. Aus meinen Präparaten kounte man die Ueberzeugung gewinnen, dass an dem atrophischen Prozesse auch der Kern Antheil nahm, indem er die regelmässigen Contouren verlor, körnig wurde und endlich an seiner Stelle nur das Kernkörperchen, das mit zerstreuten Körnern umgeben war, zurücklassend, verschwand. Was aber weiter mit dem Kernkörperchen geschah, konnte ich nicht erforschen. Ich muss hier aber hinzufügen, dass die Veränderungen im Kerne nicht immer den Veränderungen im Protoplasma entsprachen: aus den beigefügten Zeichnungen kann man sich überzeugen, dass sehr veränderte Kerne oft zu Zellen gehören, in denen kaum ein unbedeutendes Quantum Pigment sich befindet, und umgekehrt finden wir, wie es scheint, normale Kerne in vollständig atrophischen Zellenkörpern. In dieser Hiusicht stimmen meine Beobachtungen mit denen vieler vorhergehender Beobachter, welche sich mit der Untersuchung der pathologischen Prozesse in den Nervenzellen beschäftigten, nicht überein. Charcot und Gombault ${ }^{1}$ ) haben die Bemerkung gemacht, dass bei der Atrophie der Nervenzellen ein hervorragendes Zeichen ihres Zerfalls der Umstand sei, dass der Kern lange Zeit seinen normalen Charakter beibehält. Dasselbe setzen $\operatorname{Erb}^{2}$ ), Hoffmann ${ }^{3}$ ), Martineau ${ }^{4}$ ), Adler ${ }^{5}$ ), Obersteiner ${ }^{6}$ ) u. v. A. voraus. Als ich die im Rückenmark unter Einfluss acuter Arsenik-, Quecksilber- und Bleivergiftungen vorkommenden Veränderungen erforschte, habe ich oft auch ein fast vollständiges Verschwinden der Zelle, während der Kern gleichsam normal blieb, beobachtet ${ }^{7}$ ).

Ueberhaupt sind unsere Kenntnisse über das Endschicksal des Kernes der Nervenzellen beim Zerfallen der letzteren sehr

1) Archives de physiologie normale et patbologique. 1875. V. 2.

3) Ziemssen's Handbuch Bd. XI.

3) Vierteljahresschrift. f. Psych. 1866. S. 52.

4) Union médicale. 1874. No. 30 .

5) Archiv f. Psych. 1875. Bd. 5. H. 2.

6) Op. cit.

7) Beiträge zur Kenntniss der acuten toxischen Myelitis. Russisch. 1882. 
mangelhaft. Die meisten Verfasser sehen den Kern als sehr standhaft und widerspänstig, keinen Veränderungen unterliegend an oder erwähnen nur oberlächlich seine Vernichtung. Sehr wenige Autoren zeichnen diese Vernichtung mehr speciell. So z. B. erwähnt Frommann (op. cit.) in der Beschreibung der Veränderungen der Nervenzellen bei Sclerosis disseminata, dass in einigen Zellen veränderte Kerne sichtbar sind, - was jedoch verhältnissmässig selten vorkomme: solche Kerne seien gewöhnlich so trübe, dass man darin weder das Kernkörperchen, noch die Kernscheide erkennen könne; in anderen Fällen seien die Contouren des Kernes unvollständig, gleichsam aus einer Reihe zerstreuter Körner, deren Durchmesser der Stärke der Scheide gleich sei, bestehend; oder auch die ganze Scheide, in sehr kleine, an einander liegende Körner zerfallend, bilde um den Kern wie eine trübe Hülle, in welcher man manchmal kleine Vacuolen bemerke. Hayern ${ }^{1}$ ) zeigt in der Beschreibung des anatomischen Bildes einer acuten diffusen Myelitis, dass die anfangs deutlich sichtbaren Zellenkerne später zusammenschrumpien und atrophisch werden. Tschisch ${ }^{2}$ ) fand bei Untersuchung des Rückenmarks vergifteter Hunde, dass bei der Vergiftung durch Morphin und Atropin die Kerne der Nervenzellen bedeutend verändert waren: es zeigten sich namentlich grobe Körner in denselben, was er bei Vergiftung durch Arg. nitric. und Kal. brom. niemals bemerkt hat. Auf ähnliche Veränderungen in den Kernen, wie ich sie oben beschrieben habe, wies vor Kurzem Friedmann ${ }^{3}$ ) bei einer acuten Gehirnentzündung hin.

Aus den angegebenen Thatsachen ist ersichtlich, dass in den Nervenzellen zweierlei Prozesse stattfinden können: in dem einen werden die Kerne erst am Ende und zwar nicht erheblich betheiligt, im anderen unterliegen sie schon sehr früh Veränderungen, während das Protoplasma noch keine bemerkbaren Störungen zeigt. In usserem Falle konnte man diese beiden Prozesse constatiren; es ist hier daher eigentlich von einem wirklichen Unterschiede ihres Charakters nicht die Rede. Die natürlichste

1) Archives de physiol. 1874. T. 1.

2). Ueber die Veränderungen des Rückenmarks bei Vergiftungen dureh Morphin etc. 1883. Russisch.

3) Op. cit. 
Annahme ist wohl die, dass die grössore oder geringere Widerstándsfähigkeit des Kernes gegen schädliche Einfliasse von der Individualität der Ernährungsverhältnisse, in welchen die einzelnen Nervenzellen sich befinden, abhängt.

Die Analyse der oben beschriebenen Veränderungen der Nervenzellen und Fasern lehrt überzengend, dass wir es mit . pathologischen Erscheinungen an denselben zu thun haben. Was für eine Art von krankhaftem Prozesse hat aber die Nervenzellen befallen?

Zu allererst, glaube ich, müssen wir feststellen, dass hier von der passiven Eigenschaft, welche Schaffer diesen Erscheinungen zuschreibt, keine Rede sein kann. In der That waren die pericellulären Räume vicht nur erweitert, sondern sogar in der ungeheuren Mehrzahl nicht deutlich erkennbar; überhaupt lagen die Nervenzellen der umgebenden Neuroglia dicht an und gleichzeitig waren sie stark mit Pigment angefültt. Es unterliegt keinem Zweifel, dass dieses Pigment sich .ohne Einfluss eines äusseren Druckes gebildet hat und nur als Resultat der Störung in der Ernährung, welche in den Zellen stattfand, hervortrat: Es wäre sogar a priori unwahrscheinlich anzunehmen, dass so zarte Elemente, wie die Nervenzellen, welche eine so wichtige Rolle im Leben des Organismus spielen, an dem stürmischen Prozesse, dessen Sitz bei der Lyssa das Centralnervensystem ist, - ein System, dessen Functionsstörungen gerade den Grund des klinischen Bildes darstellen, - keinen Antheil nehmen sollten.

Leider sind uns die pathologischen Prozesse, welche in den Nervenelementen überhaupt und in den Zellen besonders vorkommen, bis jetzt noch sehr wepig bekannt; bis jetzt hat noch die Meinung Erb's ihre Kraft, dass wir die Entzündungserscheinungen von den atrophischen genau zu trennen nicht im Stande sind. Zwar hat Tigges') Kerntheilung in den Nervenzellen angegeben, und diese als ein Zeichen des activen Entzündungsprozesses betrachtet; später beschrieb auch Hamilton ${ }^{2}$ ) einen solchen Vorgang, aber diese Beobachtungen sind bis heute noch nicht bestätigt ${ }^{3}$ ).

1) Allgem. Zeitsehr. f. Psych. 1863. Bd. XX.

2) Op. cit.

3) Nachdem Flemming seine berühmte Untersuchungsmethode angegeben hat, haben zwar auch in den letzten Zeiten Moridino und Coen die Arehiv f. pathol. Auat. Ba. 122. Eff. 1. 
Viele Pathologo-Anatomen bezeichnen einen Zustand der Nervenzelle als charakteristisch für den Entzündungsprozess: die trübe Schwellung, fügen aber hinzu, dass die trüb angeschwollene Zelle schnell weiteren Veränderungen, meistentheils von atrophischem Charakter, unterliegt.

In unserem Falle konnte man trüb geschwollene Zellen in Gegenden, welche am wenigsten von dem Prozesse betroffen. waren, wahrnehmen. Diese Thatsache, in Verbindung mit den anderen Krankheitserscheinungen, welche das Centralnervensystem betroffen haben, sprechen für die Annahme, dass die Veränderungen der Nervenzellen als entzündliche anerkannt werden müssen.

Als ich oben eine specielle Beschreibung der Resultate meiner. Untersuchung gab, habe ich angemerkt, dass die Intensität der Veränderungen der Nervenzellen in den verschiedenen Abtheilungen des Centralnervensystems sehr ungleichmässig war. Unwillkürlich stellt sich hier die Frage, ob diese Ungleichmässigkeit des Prozesses zufällig oder von gewissen Bedingungen abhängig, - ob sie so zu sagen gewissen Gesetzen unterworfen war? Die Ungleichheit der Veränderung des Centralnervensystems bei der Lyssa ist fast von allen früheren Beobachtern bemerkt worden, aber der grösste Theil derselben erklärte sie damit, dass der pathologische Prozess aus gewissen Ursachen schärfer im verlängerten Mark, dem Hauptsitz des Leidens, hervortrete und allmählich in der Richtung nach oben und unten abnehme. Weller hat bei der Untersuchung des Centralnervensystems einiger Hunde und eines Menschen gefunden, dass die Veränderungen bei dem Menschen bedeutend mehr beschränkt waren, als bei den Hunden. Beim Menschen finden sie sich überwiegend im verlängerten Mark und in der Halsanschwellung des Rüekenmarks. Laufenauer spricht in seinem erwähnten Berichte bestimmt aus, dass die Gefahr der Infection um so grösser ist, je näher dem verlängerten Mark die verwundete Stelle sich befindet, und dass zugleich der Zeitraum der Incubation verkürzt wird.

Wenn man die Resultate in unserem Falle verallgemeinern will, so stellt sich die Sache in etwas anderem Lichte dar.

Kerntheilung in Nervenzellen beobachtet, jedoch ist es, so viel mir bekannt, trotz mebrmaliger Versucbe, keinem gelungen, ibre Arbeit zu bestätigen. 
Wir haben schon bei der Beschreibung des mikroskopischen Bildes im verlängerten ,Mark gesehen, dass verschiedene Gruppen der Nervenzellen, welche in einem und demselben Durchschnitte, mithin in einer und derselben Höhe sich befinden, ungleichmässig gelitten haben; neben den sehr veränderten Kernen des XII. Nervenpaares z. B. wurde ein fast vollständiger Mangel an Veränderungen in den Zellen der Oliven u. s. w. vorgefunden.

Ausserdem erwies die Untersuchung des Rückenmarks, dass im Lendentheile die Nervenzellen stärker, als im Brusttheil, litten; im Gehirn war die Intensität des Prozesses ebenfalls ungleichmässig, ohne dass er sich allmählich, wie dies die obenerwähnten Autoren angegeben haben, vermindert hätte. Wenn man erwägt, dass die Veränderungen der Nervenzellen, die zu einer und derselben Gruppe gehören, in der Nähe der Gefässe am stärksten sind, so könnte man glauben, dass die einzelnen Zellengruppen um so stärker leiden, je reichlicher sie vom Blute benetzt werden. Die präcise Untersuchung der Schnitte hat jedoch erwiesen, dass eine solche Annahme in der Wirklichkeit nicht zutrifft. Zu demselben Schlusse müssen wir gelangen, wenn wir unsere Untersuchungen mit denen der früheren Beobachter vergleichen, von denen viele, z. B. Weller, sehr speciell die Topographie des pathologischen Prozesses beschreiben. Bei Hunden hat Weller die grössten Veränderungen im verlängerten Mark, besonders in der Gegend der Kerne der XI., X. und IX. Nervenpaare, gefunden; in der Brücke litten am meisten die Kerne der VIII., VII. und VI. Nervenpaare. In den Vierhügeln betrafen die Entzündungserscheinungen mit gleicher Kraft die ganze graue Substanz. Im Sehhügel und im gestreiften Körper waren sie schon weniger sichtbar und schwanden nach und nach in der Richtung nach vorne so; dass sie in den Hirnhemisphären (mit Ausnahme der Geruchslappen) sehr selten aufgefunden warden. Bei der Untersuchung des Centralnervensystems eines an Hydrophobie gestorbenen Menschen hat Weller eine noch mehr begrenzte Verbreitung des pathologischen Prozesses gefunden: Entzündungserscheinungen kamen in den Hirnganglien gar nicht vor, ebenso waren die Kerne der III., IV., VII. und XII. Nervenpaare ganz verschont.

Es ist genügend, diese Beschreibung mit den Veränderungen 
in unserem Falle zu vergleichen, um die Ueberzeugung zu gewinnen, wie sehr dieselben unter einander verschieden sind. Wir haben gesehen, dass die Nervenzellen am meisten in der Gegend der Kerne des XII. Paares verändert waren, während sie nach Weller fast gar keine Spur von Entzündungszeichen zeigten: Und doch, soweit es die Gefässe betrifft, bestätigen meine Präparate vollständig die Beobachtung Weller's. Auch in den einzelnen Abtheilungen der Hirnganglien habe ich hervorragende Veränderungen der Nervenzellen constatirt, während Weller hier seitens der Gefässe nichts Pathologisches gefunden hat.

Bei einer genauen Beachtung der oben beschriebenen Localisation des Prozesses kommt man jedoch unwillkürlich zu der Annahme, dass die grösste Intensität der Veränderungen in den Zellengruppen mit ihrer physiologischen Function nicht in Beziehung steht.

In Wirklichkeit kann eine solche Frage nicht mit voller Präcision gelöst werden; ein wichtiges Hindernisss hierin sind die ungenügenden Kenntnisse ars dem Gebiete der Physiologie des Centralnervensystems. Nichtsdestoweniger sind die wenigen Thatsachen, in Besitz derer wir heute schon sind, im Stande, uns, meiner Meinung nach, gewisse sehr interessante Winke zu geben.

Wir haben oben schon gesehen, dass von Nervenzellen des Rückenmarks diejenigen am meisten gelitten hatten, die in den Vorderhörnern liegen. Eben diese Zellen aber sind, nach den jetzt. Kraft habenden Ansichten, in enger Verbindung mit den Vorderwurzeln; ihre Hauptfunction ist die Bewegung. In der Gegend des verlängerten Marks, wo überall der pathologische Prozess seine höchste Stufe erreicht hatte, waren besonders diejenigen Zellen, welche die Kerne der rein motorischen Nerven bilden, verändert. In der Hirnrinde zeigten die Nervenzellen der Stirn- und Paracentrallappen bei weitem grössere Veränderungen, als die der Hinterlappen; die Mehrzahl der Physiologen sieht gerade in den ersteren die motorischer Centra.

Was nun die Hirnganglien anbelangt, so mtissen wir uns darüber noch sehr behutsam ausdrücken, da die Ansichten über dieselben sehr streitig sind. Nichtsdestoweniger wird das Augenmerk auch hier unwillkürlich auf einige Details gerichtet. Wir haben oben gesehen, dass die Kerne der vorderen Vierhügel 
mehr, als die der hinteren, verschont blieben. Viele Autoren aber bringen die ersteren mit dem Sehen in Verbindung, und von den letzteren behaupten eiuige, z. B. Ferrier, dass in ihnen Kerne zum Ausdrucke der Empfindungen, d. h. motorische, vorhanden seien: Ich werde mich nicht speciell mit den Sehhügeln beschäftigen, deren Function uns noch weniger bekannt ist. Aber, was man bis jetzt erfahren hat, genügt meiner Meinung nach, um den Schluss zu ziehen; dass in unserem Falle der pathologische Prozess mit besonderer Kraft diejenigen Nervenzellengruppen, deren Function die Bewegung ist, erfasst hat.

Den Beweis dieser Folgerung finden wir in dem klinischen Bilde des Leidens, in welchem bekanntiich verschiedene motorische Störungen, die sich anfangs durch Aufregung und dann durch Depression der motorischen Sphäre offenbaren, die grösste Rolle spielen.

Erst nach Beendigung dieser Untersuchung habe ich, Dank dem Wohlwollen des Dr. K. Schaffer, dessen letate Arbeit, welche eben den Druck verlassen hat und gleichfalls der Hundswuth gewidmet ist, erhaltẹn. In dieser Arbeit aualysirt der Verfasser, auf Grund von 12 Fällen von Hydrophobie bei Menschen, genau die Details des klinischen Bildes des Leidens; vergleicht sie mit den Resultaten der mikroskopischen Untersuchung, welche er an der Hälfte seiner Fälle vorgenommen hat, und bemüht sich auf diese Weise, eine rationelle Theorie der Krankheit aufzustellen.

Seine Grundschlüsse stellt er folgendermaassen dar:

1. Bei der Hydrophobie entwickelt sich eine acute Myelitis, an welcher die Nervenelemente einen besonderen Antheil nehmen.

2. Diese Myelitis erscheint in zweifacher Art: entweder ist die Infiltration des Gewebes sehr intensiv, ohne dass. Erscheinungen starker Nekrose vorkommen, oder die Infiltration ist weniger stark, aber es treten bedeutend verbreitete Erweichungen ein.

3. Auf Grund der Erfahrung, dass am meisten deutliche Veränderungen im Rückenmark sich auf eine gewisse Abtheilung desselben beschränken, welche genau der Stelle des Bisses ent- 
spricht, und dass sie von dort allmählich abnehmen, kann man schon histologisch den Weg, auf welchem das Gift der Hundswuth sich verbreitet, andeuten und zugleich die anatomische Grundlage der Nerventheorie angeben (K. Schaffer, Pathologie und pathol. Anatomie der Lyssa. Beiträge zur pathol. Anatomie und zur allgem. Pathologie, Bd. VII).

Wenn man nun diese Schlussfolgerungen mit den von mir erzielten vergleicht, ist es nicht schwer zu erkennen, dass, wiewohl die Ansicht Schaffer's und die meinige über die Natur des pathologischen Prozesses in den Nervenelementen bei der Lyssa übereinstimmen, sich dies von der Localisation des Prozesses nicht sagen lässt. Schaffer hält seine frühere Meinung, dass der Sitz der grössten Veränderungen das verlängerte Mark ist, nicht aufrecht. Er fand, dass für diesen Punkt derjenige Theil des Rückenmarks, in welchem die peripherischen Nerven aus der Gegend des Bisses münden, z. B. die Lendenanschwellung; wenn der Biss an der unteren Extremität stattfand, oder die Halsanschwellung, wenn die Hand gelitten hat, angesehen werden muss. Da die peripherischen Nerven Entzündungserscheinungen zeigten, so gelangte Schaffer zu dem Schluss, dass das Gift der Hundswuth durch die peripherischen Nerven verbreitet werde und eine Rückenmarkserkrankung in entsprechender Höhe hervorrufe; von da erstrecke sich der pathologische Prozess nach oben und unten. Daher erscheine anch der zuerst leidende Theil des Rückenmarks, da er sich am längsten unter dem Einflusse des Giftes befinde, bei der Section am meisten verändert.

Die Resultate meiner Untersuchung bestätigen eine solche Auffassung nicht. In unserem Falle, wie dies schon bemerkt worden, unterlag die Localisation des pathologischen Prozesses anderen Gesetzen.

\section{Erklärung der Abbildungen. Tafel I.}

Fig. 1. Zeiss Oc. 8, Obj. 2,0. Oelimmersionslinse, 1000fache Vergrösserung. Carminfärbung. Rosenkranzartig hypertrophirte Nervenfasern aus den Rückenmarksseitensträngen.

Fig. 2. Zeiss. Vergrösserung und Färbung dieselbe. Ein Theil des Querschnittes des Rückenmarkshinterstranges, welcher einige bypertrophische Axencylinder, mit Myelin bedeckt oder obne dieses, enthält. 
Fig. 3. Zeiss. Vergrösserung und Färbung dieselbe. Nervenzelle aus den .Rückenmarksvorderbörnern: in der Zelle ein bedeutendes Quantum von Pigment; im Kerne sieht man Vacuolen.

Fig. 4. Zeiss. Vergrösserung dieselbe. Pikrocarminfärbung. Nervenzelle aus dem Rückenmarksvorderhorn; an einem der Zellenpole ein bedeutendes Quantum von Pigment sichtbar, der Kern von unregelmässiger, sternförmiger Form.

Fig. 5. Zeiss Oc. 8, Obj. 4,0. 500fache Vergrösserung. Carminfärbing، Nervenzellengruppe aus dem Rückenmarksvorderhorn. Zwei Zellen in der Nähe der Gefässe so mit Pigment überfüllt, dass der Kern nicht sichtbar ist; zwei andere, vom Gefässe entfernt, ungleich weniger verändert.

Fig. 6. Zeiss. Vergrösserung und Färbung dieselbe. Nervenzellengruppe aus der Gegend des XII. Nervenpaares, zeigt verschiedene Stufen der pigmentösen Atrophie; in der unten und links liegenden Zelle ist der Kern, trotz bedeutender Veränderung des Protoplasma, erhalten.

Fig. 7. Zeiss Oc. 8, Obj. 2,0. Oelimmersionslinse. 1000fache Vergrösserung. Färbung nach Gaule's Methode. Im erweiterten pericellularen Raume befindet sich eine Nervenzelle mit Kern, in welchem rings um das Kernkörperchen herum eine Gruppe grosser Körner sichtbar ist.

Fig. 8. Zeiss. Vergrösserung und Färbung dieselbe. Nervenzelle verbältnissmässig gut erhalten, sehr veränderter Kern. 